\title{
Coexistence Of A Huge Venous Thromboembolism And Bleeding Tendency In Cytokine Release Syndrome During CAR-T Therapy
}

This article was published in the following Dove Press journal: OncoTargets and Therapy

\author{
Haiyan Liu' ${ }^{1, *}$ \\ Ye Yang $\mathbb{D}^{2, *}$ \\ Jie Jiang' \\ Xinfeng Wang' \\ Chenlu Zhang' \\ Yijing Jiang' \\ Lemin Hong (D) \\ Hongming Huang $\mathbb{D}^{\prime}$ \\ 'Department of Hematology, Affiliated \\ Hospital of Nantong University, Nantong, \\ Jiangsu 22600I, People's Republic of \\ China; ${ }^{2}$ School of Integrative Medicine, \\ Nanjing University of Chinese Medicine, \\ Nanjing, Jiangsu 210023, People's \\ Republic of China
}

*These authors contributed equally to this work
Correspondence: Hongming Huang Department of Hematology, The Affiliated Hospital of Nantong University, 20 Xisi Road, Nantong 22600I, People's Republic of China

Email hhmmmc@163.com

\begin{abstract}
Chimeric antigen receptor (CAR)-modified T cell therapy is increasingly administered for hematological malignancies. Cytokine release syndrome (CRS) is a common and severe complication of CAR-T therapy. In the present case, a 62-year-old male patient was diagnosed with relapsed and refractory multiple myeloma (RRMM). Treated with CART-CD19/BCMA therapy, his symptoms remitted, during which occasional but severe CRS associated with coagulation disorder still appeared, as evidenced by the coexistence of a huge thrombosis and bleeding tendency. Through the First Generation Sequencing, we extracted genomic DNA from the patient's peripheral blood to analyze the distribution of polymorphism at the $-572 \mathrm{C} / \mathrm{G}$ site of the promoter of IL- 6 gene. The results showed that the genotype of $-572 \mathrm{C} / \mathrm{G}$ promoter polymorphism was CC, indicating that high level of IL-6 and $-572 \mathrm{C} / \mathrm{G}$ polymorphism might be associated with the risk of thrombotic disorders. We concluded that immediate diagnosis and appropriate treatment of coagulopathy could reduce CRS-related mortality.
\end{abstract}

Keywords: chimeric antigen receptor-T therapy, cytokine release syndrome, multiple myeloma, coagulation disorder, thrombosis

\section{Introduction}

Currently, multiple myeloma (MM) can be effectively controlled with proteasome inhibitors (PI), immunomodulatory drugs (IMiDs), and autologous stem cell transplantation. Complete remission (CR) can be achieved in $>80 \%$ of newly diagnosed MM patients, and a median survival of $>10$ years in those aged $<50$ years. ${ }^{1-3}$ Even so, MM remains incurable. For example, even if treated with bortezomib plus lenalidomide, RRMM patients showed significantly reduced time-to-progression (TTP) and/or median overall survival (OS). ${ }^{4,5}$ Also, the prognosis of MM patients who relapse with the transformed pattern, may be just 10 months. ${ }^{6}$ New options are urgently needed for MM treatment.

A revolutionary therapeutic modality for cancer treatment is genetically modifying autologous $\mathrm{T}$ cells to express chimeric antigen receptors (CARs) that in turn redirect $\mathrm{T}$ cells to eliminate tumor cells. ${ }^{7-9}$ This strategy has reaped good clinical outcomes in treating RRMM. BCMA and CD19 have been certified as ideal targets in CAR-T therapy for RRMM. ${ }^{9,10}$ Besides, this therapy often triggers cytokine release syndrome (CRS), an event sometimes extremely severe. ${ }^{11}$ Here we report a patient who developed serious CRS and coagulation disorder manifested with a huge thrombus in the femoral vein and a bleeding tendency. Tocilizumab was used to control his condition. 
The 62-year-old male patient was diagnosed with MM (Isotype IgG- $\lambda$; DS stage IIIB; R-ISS III) in April 2016. After the patient received a regimen of bortezomib, doxorubicin, and dexamethasone (PAD) for four cycles, CR was achieved. However, the $\lambda$ serum-free light chain remained high. Then, he was treated with another two cycles of PAD regimen and autologous hemopoietic stem cell transplantation (Auto-HSCT). Half a year later, his lower limbs and hips suffered pain serious enough to prevent him from walking, despite that bortezomib was still being used. A positron emission tomography (PET)/CT scan demonstrated multiple inguinal lymph nodes, subcutaneous tissue nodules, and localized bone destruction. Mass biopsy of the left piriformis showed massive infiltration of malignant plasma cells, suggestive of extramedullary relapse. The patient was treated with BIRD (lenalidomide, clarithromycin, and dexamethasone) and DECP (cisplatin, etoposide, isocyclophosphamide, and dexamethasone) for five cycles. Inguinal lymph nodes shrunk after chemotherapy, but swelled soon. Later, he achieved only slight remission. Unfortunately, he developed nausea, jet vomiting, and gradually blurred binocular vision until blindness in January 2018. Lumbar puncture revealed a large number of $\mathrm{CD}_{138^{+}}$myeloma cells in his cerebrospinal fluid (CSF). He was, therefore, recruited into our CAR-T clinical research.

In this research, peripheral-blood mononuclear cells were collected. While during the generation of CAR-T cells, B-ultrasound showed a lymph node $\left(106^{*} 65 \mathrm{~mm}\right)$ in the left groin. Then, the patient was re-treated with DECP therapy to reduce tumor burden and the swollen lymph node became smaller. After the administration of regimen FC, the patient received consecutive infusions of CD19-CAR-T cells and BCMA-CAR-T cells (Figure 1). Within three days after the infusion of CAR-T cells, the patient gradually developed fever, hypotension, hypoxemia, and sinus tachycardia. Subsequently treated with antipyretics, oxygen and fluid replacement, the patient's vital signs got stabilized. However, on the morning of D5, his body temperature rose to $39^{\circ} \mathrm{C}$ (Figure 2A). His left lower extremity suddenly swelled with pain, exceeding his right thigh by $12.3 \mathrm{~cm}$ in circumference (Figure 2B). His vascular B-ultrasound showed a filling defect, suggestive of the possible embolization. His platelet count decreased to $25 \times 10^{9} / \mathrm{L}$. Therefore, a rivaroxaban $(15 \mathrm{mg}$, bid) was administered for anticoagulation. The swelling in his left leg did not relieve, but worsened on D6. At the same time, his prothrombin time (PT) was increased significantly to $29.4 \mathrm{~S}$, activated partial thromboplastin time (APTT) to $94.8 \mathrm{~S}$, and body temperature to $39.2^{\circ} \mathrm{C}$ (Figure 2C, Table 1). The risk of bleeding elevated, an outcome contradictory to the goal of thrombosis therapy. Continuous anticoagulative efforts were offered, synchronizing with the infusion of cryoprecipitate and plasma to supply the coagulation factors.

On D6, serum cytokine interleukin-6 (IL-6) jumped quickly to $1180.7 \mathrm{pg} / \mathrm{mL}$, and TNF- $\alpha$, IFN- $\gamma$, and IL-10 also increased rapidly. In this condition, tocilizumab $(8 \mathrm{mg} / \mathrm{kg})$ was given immediately. But on D7, the swelling in his left lower extremity showed aggravation. Thrombosis was considered because vascular ultrasound showed a local thrombus $>10 \mathrm{~cm}$ long in the left femoral vein. The same dose of tocilizumab was used on D8 as IL-6 reached the peak $(11,403.2 \mathrm{pg} / \mathrm{mL}$ ) (Figure 2D, Table 1). Finally, the swelling of the left lower extremity began to subside. Vascular ultrasound detected no thrombus on D16. The clinical indicators, including body temperature, blood pressure, CRP and coagulation factor, returned gradually to normal; the levels of cytokines, including IL-6, dropped rapidly. The patient in a good condition was discharged on D30.

On D60, MRI showed the mass in the left piriformis shrunk significantly (Figure 3). A lumbar puncture revealed no cancer cells in the patient's cerebrospinal fluid. The patient recovered his vision earlier than expected and could walk unaided. This recovery lasted for half a year, during which MM relapsed in the central nervous system (CNS). He gave up further treatment due to financial reasons and died two months later.

\section{Discussion And Literature Review}

It is thorny to treat extramedullary myeloma (EMM) that occurs in $10-30 \%$ of patients with relapsed myeloma. ${ }^{12}$ The

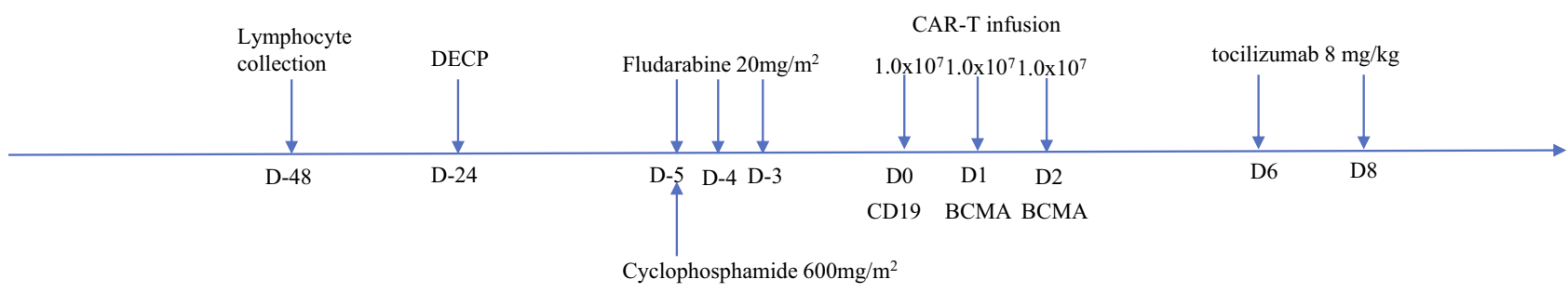

Figure I Mononuclear cells were collected from the peripheral blood on D-48; DECP chemotherapy on D-24; FC (fludarabine, 20 mg/m ${ }^{2}$ per day on D-5, D-4, and D-3; cyclophosphamide $600 \mathrm{mg} / \mathrm{m}^{2}$ on D-5); CAR-T cells were infused into the patient (CDI9 on D0 and BCMA on DI to D2); Tocilizumab (8 mg/kg) on D6 and D8. 

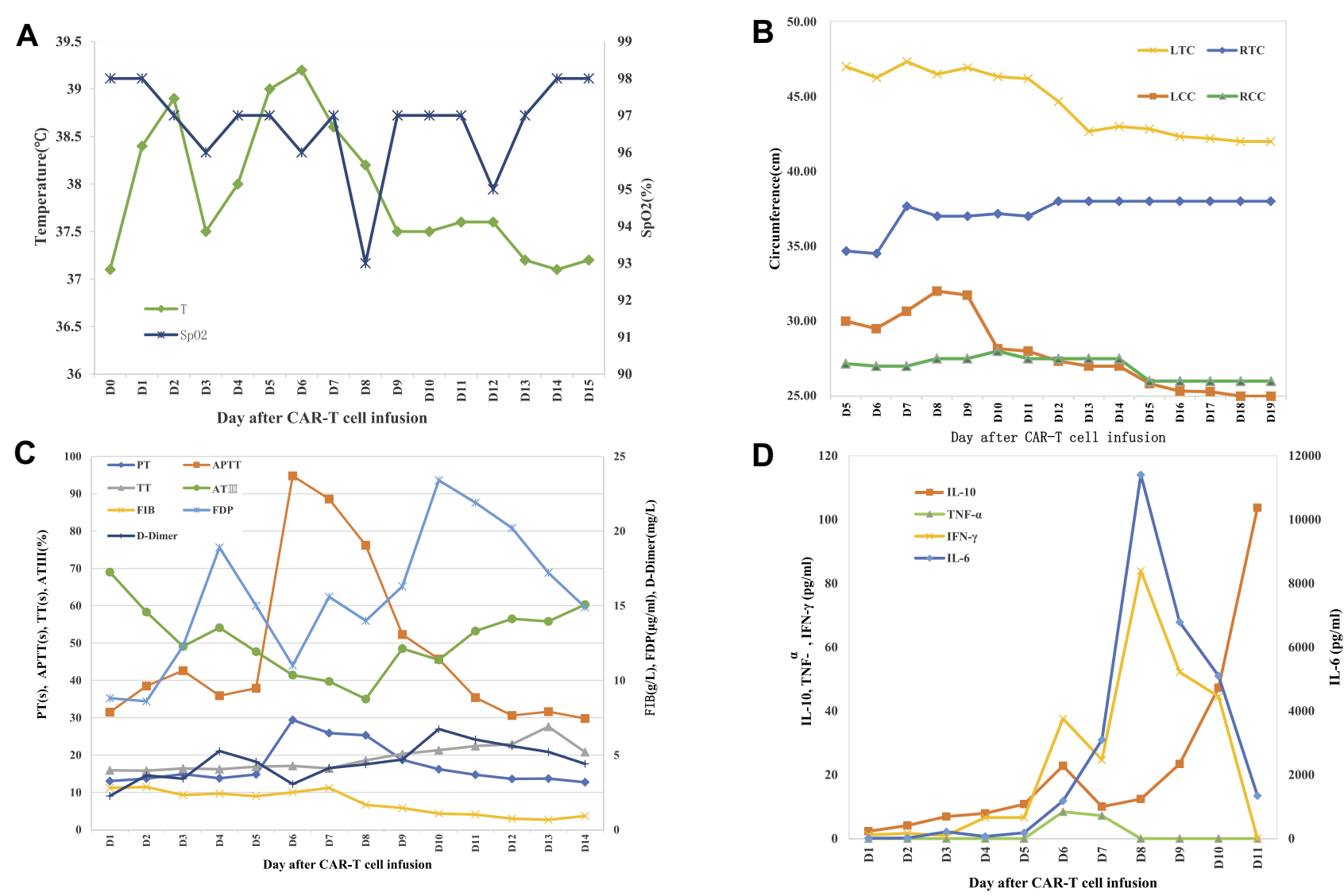

Figure 2 (A) Body temperature changes after CAR-T cell infusion, with a maximum temperature on each day and the changes in SpO ${ }_{2}$; (B) The left thigh circumference (LTC), right thigh circumference (RTC), left calf circumference (LCC), and right calf circumference (RCC) were measured every day after thrombosis was formed; (C) The levels of PT, APTT, thrombin time (TT), D-Dimer, fibrinogen (FIB), fibrin degradation products (FDP), and antithrombin III (ATIII) during the CRS changed with the levels of C-reactive protein (CRP), interleukin-6 (IL-6), etc. After treatment with tocilizumab, both coagulative and CRS indicators dropped obviously; (D) Inflammatory cytokine levels after CAR-T cell infusion (IL-6, IL-I0, TNF- $\alpha$, and IFN- $\gamma$ ); IL-6 level ( I I,403.2 pg/mL) peaked on D8.

CNS involvement, observed in only $1 \%$ of MM patients, ${ }^{13}$ has an extremely poor prognosis, with a median overall survival of $<6$ months. ${ }^{14,15}$ Our EMM patient had undergone multiple conventional chemotherapies, including use of lenalidomide capable of crossing the blood-brain barrier (BBB); ${ }^{16}$ but his condition deteriorated till the occurrence of binocular

Table I Predominant CRS Indicators After CAR-T Therapy

\begin{tabular}{|l|l|l|l|l|l|l|l|l|l|l|l|l|l|l|l|}
\hline Day & $\mathbf{1}$ & $\mathbf{2}$ & $\mathbf{3}$ & $\mathbf{4}$ & $\mathbf{5}$ & $\mathbf{6}$ & $\mathbf{7}$ & $\mathbf{8}$ & $\mathbf{9}$ & $\mathbf{1 0}$ & $\mathbf{I I}$ & $\mathbf{1 2}$ & $\mathbf{1 3}$ & $\mathbf{1 4}$ & $\mathbf{1 5}$ \\
\hline T $\left({ }^{\circ} \mathrm{C}\right)$ & 38.4 & 38.9 & 37.5 & 38 & 39 & 39.2 & 38.6 & 38.2 & 37.5 & 37.5 & 37.6 & 37.6 & 37.2 & 37.1 & 36.2 \\
CRP $(\mathrm{mg} / \mathrm{L})$ & 16.8 & 31.3 & 77.4 & 68.7 & 80.3 & 138 & 313 & 113 & 39.8 & 28.5 & 24.5 & - & 13.4 & - & 9.26 \\
IL-I0 $(\mathrm{pg} / \mathrm{mL})$ & 2.3 & 4.1 & 6.9 & 7.9 & 10.8 & 22.8 & 10 & 12.4 & 23.4 & 47.3 & 103.7 & - & 75.4 & - & 36.8 \\
TNF- $\alpha(\mathrm{pg} / \mathrm{mL})$ & 0 & 0 & 0 & 0 & 0 & 8.4 & 7.2 & 0 & 0 & 0 & 0 & - & 0 & - & 0 \\
IFN- $\gamma(\mathrm{pg} / \mathrm{mL})$ & 1.2 & 1.6 & 1 & 6.6 & 6.6 & 37.6 & 24.7 & 83.9 & 52.2 & 44.5 & 0 & - & 0 & - & 0 \\
IL-6 $(\mathrm{pg} / \mathrm{mL})$ & 12.5 & 16.4 & 213.4 & 66.3 & 181.5 & 1180.7 & 3098 & 11.403 .2 & 6784.4 & 5098.7 & 1343.6 & - & 876 & - & 431.5 \\
PT $(\mathrm{s})$ & 13 & 13.7 & 14.9 & 13.8 & 14.8 & 29.4 & 25.9 & 25.3 & 18.7 & 16.2 & 14.7 & - & 13.7 & - & 12.9 \\
APTT $(\mathrm{s})$ & 31.5 & 38.5 & 42.6 & 35.9 & 37.9 & 94.8 & 88.6 & 76.2 & 52.3 & 45.7 & 35.4 & - & 31.6 & - & 31 \\
TT $(\mathrm{s})$ & 15.9 & 15.8 & 16.4 & 16.2 & 16.9 & 17.1 & 16.4 & 18.6 & 20.3 & 21.3 & 22.4 & - & 27.6 & - & 20.7 \\
AT III $(\%)$ & 69 & 58.3 & 49.1 & 54.1 & 47.7 & 41.4 & 39.7 & 35 & 48.5 & 45.5 & 53.2 & - & 55.8 & - & 63.3 \\
FIB $(\mathrm{g} / \mathrm{L})$ & 2.8 & 2.86 & 2.32 & 2.42 & 2.25 & 2.5 & 2.8 & 1.66 & 1.45 & 1.08 & 1.01 & - & 0.66 & - & 1.09 \\
FDP $(\mu \mathrm{g} / \mathrm{mL})$ & 8.8 & 8.6 & 12.3 & 18.9 & 15 & 11 & 15.6 & 14 & 16.3 & 23.4 & 21.9 & - & 17.2 & - & 10.7 \\
DDimer $(\mathrm{mg} / \mathrm{L})$ & 2.26 & 3.64 & 3.41 & 5.27 & 4.55 & 3.05 & 4.13 & 4.38 & 4.7 & 6.74 & 6.03 & - & 5.21 & - & 3.23 \\
\hline
\end{tabular}




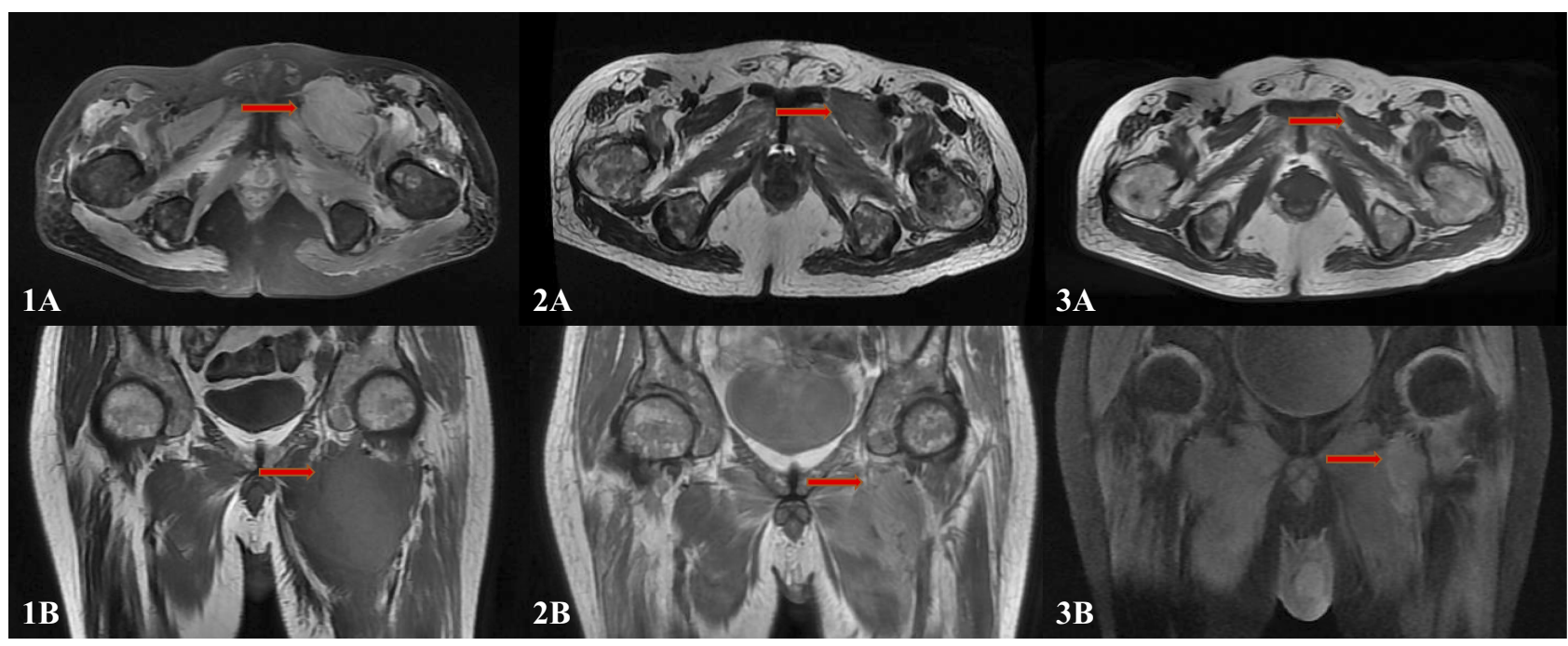

extramedullary relapse

one month after CAR-T

two months after CAR-T

Figure 3 MRI results before and after CAR-T treatment. The mass in the left piriformis region was significantly smaller after CAR-T treatment. IA and IB (extramedullary relapse); 2A and 2B (one month after CAR-T); 3A and 3B (two months after CAR-T).

blindness. CAR-T therapy has been newly developed to counter RRMM. BCMA has been proven to be a golden remedy for MM, with strong effect and weak toxicity. ${ }^{17,18}$ Though CD19 is not expressed in plasma cells, it can still target MM stem cells ${ }^{19}$ and minor $\mathrm{CD}_{1} 9^{+}$populations in an otherwise $\mathrm{CD} 19^{-}$ myeloma harbored enhanced myeloma-propagating capability. ${ }^{9}$ A recent clinical trial showed that autologous stem-cell transplantation (ASCT), followed by treatment with anti-CD19 CAR-T cells, led to a progression-free survival (PFS) of $<1$ year, despite that only merely $0.05 \%$ CD19 were expressed in the plasma cells. ${ }^{8}$ A pre-clinical research in China (NCT03455972) is recruiting patients to test the efficacy of a sequential CD19/BCMA CAR-T after ASCT. In the present case, combination of anti-CD19 with anti-BCMA CAR was effective and safe.
During the treatment, the patient suffered from severe side effects. According to CRS grading standards, Neelapu et $\mathrm{al}^{20}$ suggest a graded treatment of CRS. Primary therapy for grade 1 CRS, and anti-interleukin-6 medications such as tocilizumab are recommended for patients with grade $2 \mathrm{CRS}$, and can be reused if needed. ${ }^{21}$ After the infusion of CAR-T cells, our patient gradually developed grade $1 \mathrm{CRS}$, signs and symptoms improved after primary therapy. However, from D5 he was diagnosed with grade $2 \mathrm{CRS}$ and fatal coagulation disorders. ${ }^{21}$ The thromboembolism (VTE) in his left femoral vein caused intensive swelling and severe pain on D5 to D8. The levels of D-Dimer, fibrin degradation products (FDPs), PT, and APTT began to rise on D5, peaked on D6 to D8, then decreased gradually after two doses of tocilizumab on D6 and D8. These changes were also found in the indicators of CRS, such

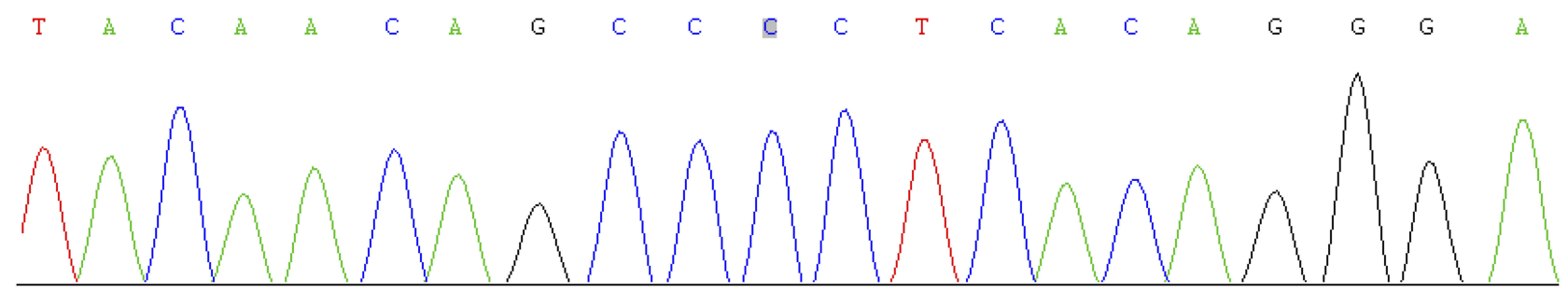

Figure 4 The result of IL-6 gene First Generation Sequencing showed that thegenotype of $-572 \mathrm{C} / \mathrm{G}$ promoter polymorphisms was CC. The sequence is GGAACCGGTGAGACGTGGCAGAGCCACGCGGTGGCAAAAAGGAGTCACACACTCCACCTGGAGACGCCTTGAAGTAACTGCACGAAATTTGAGGGTGGCCAGGC AGTTCTACAACAGCCCCTCACAGGGAGAGCCAGAACACAGAAGAACTCAGATGACTGGTAGTATTACCTTCTTCATAATCCCAGGCTTGGGGGGCTGCGATGGAGT CAGAGGAAACTCAGTTCAGAACATCTTTGGTTTTTACAAATACAAATTAACTGGAACGCTAAATTCTAGCCTGTTAATCGGTTCACTGAAAATAATTCGGGCCGGTGCC ACGGGTTGCCCGTAGCCGGAGCACCCCTCACAGCGGAGGGGCGGAAAATTGGGAAAACTACCCGCGTCGTTCTCCTTGGCTGCTGCAGATGTGAGCGCAATCGG GAGAGGTGAGTGGGAATATAAAACGAGGAGGGGGGAAAATAGCCCTTCATA. 
as C-reactive protein (CRP), IFN- $\gamma$, IL-6, IL-10 and TNF- $\alpha$, indicating that the thrombus changed with the degree of CRS. In addition, We generally do not use tocilizumab in advance, in order to prevent the application of tocilizumab from covering the condition, obstructing the observation of the actual situation of the disease. Our findings were similar to what had been previously reported about the hemorrhagic coagulation abnormalities in the CRS caused by immunotherapy. ${ }^{22,23}$ However, VTE during CRS has never been reported.

Many studies have demonstrated that patients with hemopathy are at high risk of VTE, ${ }^{24,25}$ a condition associated with tumor aggression and poor prognosis. ${ }^{26}$ The pathogenesis of the thromboembolism in hematological malignancies is multifactorial, ${ }^{27}$ including prothrombotic factors produced by tumor cells, therapeutic agents and infectious complications. Laboratory tests ruled out the hereditary factors in this patient. His VTE displayed no clinical manifestations before CAR-T therapy. Low velocity of local blood flow (caused by lymph node enlargement in groin area) and high viscosity (caused by hyperglobulinemia) might be two risk factors for thrombosis. However, the lymph node was significantly reduced after chemotherapy with DECP on D-24 and FC on D-5. Therefore, the left inguinal lymph node compression was not obvious in the CAR-T therapy. The underlying mechanism may be that in the microenvironment, amplified CAR-T cells attacked tumor cells, produced large amounts of cytokines, activated the exogenous coagulation pathway or directly activated factor X, and ended up with DIC-like syndrome with significantly prolonged PT and APTT. ${ }^{28}$

In addition, numerous studies showed that levels of IL-6 and CRP increased in some patients with VTE. ${ }^{29-31}$ In the present case, on D5 to D8 after the CAR-T treatment, the VTE grew as the levels of IL- 6 and CRP rose (even to the peak), but disappeared after being treated with tocilizumab, indicating that VTE was associated with the elevated IL-6 level. Besides, some researchers found a significant difference in the $-572 \mathrm{C} / \mathrm{G}$ promoter polymorphism of IL- 6 gene between VTE group and control group; the CC homozygotes of the IL-6 $-572 \mathrm{C} / \mathrm{G}$ were independent risk factors for VTE. $^{32-34}$ With the First Generation Sequencing, we extracted genomic DNA from the patient's peripheral blood to analyze the distribution of polymorphisms, finding that the genotype of $-572 \mathrm{C} / \mathrm{G}$ promoter polymorphisms was CC (Figure 4). We considered that VTE was associated with the elevation of IL- 6 and $-572 \mathrm{C} / \mathrm{G}$ polymorphisms.

Globally, this case for the first time reported the coexistence of CRS-related VTE and severe bleeding tendency.
Closely monitoring inflammatory cytokines could guarantee the efficacy of CAR-T therapy.

\section{Ethics Approval And Consent To Participate}

This study was approved by the ethics committee of the Affiliated Hospital of Nantong University. The patient agreed and submitted a written informed consent to allow publication of this report and the accompanying images. Institutional approval was not required to publish this manuscript.

\section{Acknowledgments}

The authors gratefully thank Xiaojian Zhu of Tongji Hospital Affiliated to Tongji Medical College, Huazhong University of Science and Technology, for his fair advice; thank Dan Guo of Affiliated Hospital of Nantong University, for her contribution during major revision; thank Shanghai UnicarTherapy Bio-medicine Technology Co.,Ltd for supplying CAR-T CD19 cells and CAR-T CD BCMA cells.

\section{Disclosure}

The authors report no conflicts of interest in this work.

\section{References}

1. Bianchi G, Ghobrial IM. Biological and clinical implications of clonal heterogeneity and clonal evolution in multiple myeloma. Curr Cancer Ther Rev. 2014;10(2):70-79.

2. Landgren O, Iskander K. Modern multiple myeloma therapy: deep, sustained treatment response and good clinical outcomes. J Intern Med. 2017;281(4):365-382.

3. Landgren O, Owen RG. Better therapy requires better response evaluation: paving the way for minimal residual disease testing for every myeloma patient. Cytometry B Clin Cytom. 2016;90(1):14-20.

4. Morgan G, Palumbo A, Dhanasiri S, et al. Overall survival of relapsed and refractory multiple myeloma patients after adjusting for crossover in the MM-003 trial for pomalidomide plus low-dose dexamethasone. Br J Haematol. 2015;168(6):820-823.

5. Offidani M, Corvatta L, Maracci L, et al. Efficacy and tolerability of bendamustine, bortezomib and dexamethasone in patients with relapsed-refractory multiple myeloma: a phase II study. Blood Cancer J. 2013;3:e162.

6. Ahn JS, Jung SH, Yang DH, et al. Patterns of relapse or progression after bortezomib-based salvage therapy in patients with relapsed/ refractory multiple myeloma. Clin Lymphoma Myeloma Leuk. 2014;14(5):389-394.

7. Cohen AD. CAR T cells and other cellular therapies for multiple myeloma: 2018 update. Am Soc Clin Oncol Educ Book. 2018;38:e6e615.

8. Garfall AL, Maus MV, Hwang WT, et al. Chimeric antigen receptor T cells against CD19 for multiple myeloma. $N$ Engl J Med. 2015;373 (11): $1040-1047$

9. Garfall AL, Stadtmauer EA, Hwang WT, et al. Anti-CD19 CAR T cells with high-dose melphalan and autologous stem cell transplantation for refractory multiple myeloma. JCI Insight. 2018;3:8. 
10. Zhao WH, Liu J, Wang BY, et al. A phase 1, open-label study of LCAR-B38M, a chimeric antigen receptor $\mathrm{T}$ cell therapy directed against $\mathrm{B}$ cell maturation antigen, in patients with relapsed or refractory multiple myeloma. J Hematol Oncol. 2018;11(1):141.

11. Maude SL, Barrett D, Teachey DT, Grupp SA. Managing cytokine release syndrome associated with novel $\mathrm{T}$ cell-engaging therapies. Cancer J. 2014;20(2):119-122.

12. Varga C, Xie W, Laubach J, et al. Development of extramedullary myeloma in the era of novel agents: no evidence of increased risk with lenalidomide-bortezomib combinations. $\mathrm{Br} \quad \mathrm{J}$ Haematol. 2015;169(6):843-850.

13. Nieuwenhuizen L, Biesma DH. Central nervous system myelomatosis: review of the literature. Eur J Haematol. 2008;80(1):1-9.

14. Katodritou E, Terpos E, Kastritis E, et al. Lack of survival improvement with novel anti-myeloma agents for patients with multiple myeloma and central nervous system involvement: the Greek myeloma study group experience. Ann Hematol. 2015;94(12):2033-2042.

15. Touzeau C, Moreau P. How I treat extramedullary myeloma. Blood. 2016;127(8):971-976.

16. Muscal JA, Sun Y, Nuchtern JG, et al. Plasma and cerebrospinal fluid pharmacokinetics of thalidomide and lenalidomide in nonhuman primates. Cancer Chemother Pharmacol. 2012;69(4):943-947.

17. Carpenter RO, Evbuomwan MO, Pittaluga S, et al. B-cell maturation antigen is a promising target for adoptive T-cell therapy of multiple myeloma. Clin Cancer Res. 2013;19(8):2048-2060.

18. Novak AJ, Darce JR, Arendt BK, et al. Expression of BCMA, TACI, and BAFF-R in multiple myeloma: a mechanism for growth and survival. Blood. 2004;103(2):689-694.

19. Mikkilineni L, Kochenderfer JN. Chimeric antigen receptor T-cell therapies for multiple myeloma. Blood. 2017;130(24):2594-2602.

20. Neelapu SS, Tummala S, Kebriaei $P$, et al. Chimeric antigen receptor T-cell therapy - assessment and management of toxicities. Nat Rev Clin Oncol. 2018;15(1):47-62.

21. Erratum; Lee DW, Gardner R, Porter DL, et al. Current concepts in the diagnosis and management of cytokine release syndrome. Blood. 2014;124(2):188-195. doi:10.1182/blood-2014-05-552729.

22. Suntharalingam G, Perry MR, Ward S, et al. Cytokine storm in a phase 1 trial of the anti-CD28 monoclonal antibody TGN1412. $N$ Engl J Med. 2006;355(10):1018-1028.
23. Mei $\mathrm{H}$, Jiang $\mathrm{H}, \mathrm{Wu} \mathrm{Y}$, et al. Neurological toxicities and coagulation disorders in the cytokine release syndrome during CAR-T therapy. $\mathrm{Br}$ J Haematol. 2018;181(5):689-692.

24. Ziegler S, Sperr WR, Knöbl P, et al. Symptomatic venous thromboembolism in acute leukemia. Incidence, risk factors, and impact on prognosis. Thromb Res. 2005;115(1-2):59-64.

25. De Stefano V, Sorà F, Rossi E, et al. The risk of thrombosis in patients with acute leukemia: occurrence of thrombosis at diagnosis and during treatment. J Thromb Haemost. 2005;3(9):1985-1992. doi:10.1111/j.1538-7836.2005.01467.x

26. Castelli R, Ferrari B, Cortelezzi A, Guariglia A. Thromboembolic complications in malignant haematological disorders. Curr Vasc Pharmacol. 2010;8(4):482-494.

27. Falanga A, Russo L, Milesi V, Vignoli A. Mechanisms and risk factors of thrombosis in cancer. Crit Rev Oncol Hematol. 2017;118:79-83.

28. Magnus N, D'Asti E, Meehan B, Garnier D, Rak J. Oncogenes and the coagulation system-forces that modulate dormant and aggressive states in cancer. Thromb Res. 2014;133(Suppl 2):S1-9.

29. Beckers MM, Ruven HJ, Haas FJ, et al. Single nucleotide polymorphisms in inflammation-related genes are associated with venous thromboembolism. Eur J Intern Med. 2010;21(4):289-292.

30. Zacho J, Tybjaerg-Hansen A, Nordestgaard BG. C-reactive protein and risk of venous thromboembolism in the general population. Arterioscler Thromb Vasc Biol. 2010;30(8):1672-1678.

31. van Aken BE, Den Heijer M, Bos GM, van Deventer SJ, Reitsma PH. Recurrent venous thrombosis and markers of inflammation. Thromb Haemost. 2000;83(4):536-539.

32. Ren H, Zhang Y, Yao Y, et al. Association between the interleukin-6 genetic polymorphism $174 \mathrm{G} / \mathrm{C}$ and thrombosis disorder risk: meta-analysis of 10,549 cases and 19,316 controls. Medicine (Baltimore). 2016;95(27): e4030.

33. Mahemuti A, Abudureheman K, Aihemaiti X, et al. Association of interleukin-6 and C-reactive protein genetic polymorphisms levels with venous thromboembolism. Chin Med J (Engl). 2012;125(22):3997-4002.

34. Mälarstig A, Wallentin L, Siegbahn A. Genetic variation in the interleukin- 6 gene in relation to risk and outcomes in acute coronary syndrome. Thromb Res. 2007;119(4):467-473.
OncoTargets and Therapy

\section{Publish your work in this journal}

OncoTargets and Therapy is an international, peer-reviewed, open access journal focusing on the pathological basis of all cancers, potential targets for therapy and treatment protocols employed to improve the management of cancer patients. The journal also focuses on the impact of management programs and new therapeutic agents and protocols on patient perspectives such as quality of life, adherence and satisfaction. The manuscript management system is completely online and includes a very quick and fair peer-review system, which is all easy to use. Visit http://www.dovepress.com/ testimonials.php to read real quotes from published authors. 